\title{
Plyometric Training for Young Male Field Hockey Players
}

Vijaya Krishnan

MGM College of Physiotherapy, Navi Mumbai, victoryv2@yahoo.co.in

Twincy Rajawadha

twincyrajawadha95@gmail.com

Follow this and additional works at: https://nsuworks.nova.edu/ijahsp

Part of the Physical Therapy Commons, and the Sports Sciences Commons

\section{Recommended Citation}

Krishnan V, Rajawadha T. Plyometric Training for Young Male Field Hockey Players. The Internet Journal of Allied Health Sciences and Practice. 2020 Jan 01;18(3), Article 8.

This Manuscript is brought to you for free and open access by the College of Health Care Sciences at NSUWorks. It has been accepted for inclusion in Internet Journal of Allied Health Sciences and Practice by an authorized editor of NSUWorks. For more information, please contact nsuworks@nova.edu. 


\title{
Plyometric Training for Young Male Field Hockey Players
}

\begin{abstract}
Background: Field hockey is a team sport requiring a combination of skill set to enhance a player's performance. Power, Fitness and Agility are few such basic parameters. Newer training protocols are constantly explored to achieve the desired effect and plyometrics is one such method. It is also important to know how long lasting the effects of training are. Methodology: Fifty (50) male field hockey players at interschool and zonal level participated in the study. These sports specific parameters - lower limb power, fitness level and agility - were tested using the vertical jump test, 40-metre sprint test, and shuttle cross pick up test respectively. Plyometric training was supervised for 6 weeks and the values were recorded at baseline, $3^{\text {rd }}$ week, and $6^{\text {th }}$ week. The training was stopped after six weeks and the post cessation values on the $8^{\text {th }}$ week were recorded. Results: Plyometric training showed statistically significant improvement in all the test parameters throughout the 6 weeks. The effects of training post cessation of the plyometric regimen also were statistically significant after two weeks. Conclusion: Plyometric training was effective in improving the lower limb power, fitness level, and agility level. Long lasting effects of training were also noted. Coaches will find it a very effective protocol and one of the ideal methods to enhance player performance.
\end{abstract}

\section{Author Bio(s)}

Dr. Vijaya Krishnan (PT), is an Assistant Professor at the MGM College of Physiotherapy, Navi Mumbai. She aspires to be an asset to the ever growing field of physiotherapy.

Dr. Twincy Rajawadha (PT), is a Physiotherapy Graduate eager to explore the world of physiotherapy with dedication.

\section{Acknowledgements}

We would like to express our gratitude towards our College Principal and all the participants of the study. 


\title{
IIJAHSP \\ The Internet Journal of Allied Health Sciences and Practice \\ Dedicated to allied health professional practice and education
}

Vol. 18 No. 3 ISSN 1540-580X

\section{Plyometric Training for Young Male Field Hockey Players}

\author{
Vijaya Krishnan \\ Twincy Rajawadha \\ MGM College of Physiotherapy \\ India
}

\begin{abstract}
Background: Field hockey is a team sport requiring a combination of skill set to enhance a player's performance. Power, Fitness and Agility are few such basic parameters. Newer training protocols are constantly explored to achieve the desired effect and plyometrics is one such method. It is also important to know how long lasting the effects of training are. Methodology: Fifty (50) male field hockey players at interschool and zonal level participated in the study. These sports specific parameters - lower limb power, fitness level and agility - were tested using the vertical jump test, 40-metre sprint test, and shuttle cross pick up test respectively. Plyometric training was supervised for 6 weeks and the values were recorded at baseline, $3^{\text {rd }}$ week, and $6^{\text {th }}$ week. The training was stopped after six weeks and the post cessation values on the $8^{\text {th }}$ week were recorded. Results: Plyometric training showed statistically significant improvement in all the test parameters throughout the 6 weeks. The effects of training post cessation of the plyometric regimen also were statistically significant after two weeks. Conclusion: Plyometric training was effective in improving the lower limb power, fitness level, and agility level. Long lasting effects of training were also noted. Coaches will find it a very effective protocol and one of the ideal methods to enhance player performance.
\end{abstract}

Key words: hockey, plyometrics, lower limb power, fitness, agility, fatigue. 


\section{INTRODUCTION}

Being an intermittent endurance sport, field hockey requires an amalgamation of extraordinary aerobic fitness, anaerobic power, and speed and agility, in addition to optimal skills. It requires continuous dribbling of the ball, quick changes of direction, walking and sprinting throughout the competitive game where players cover the entire field during an attack, and exquisite defence tactics. Appropriate training and continual monitoring are required to achieve peak player performance and foster general health status. Newer and more effective training protocols must be included by the coaches and health experts to improve the game, to enhance the player performance, and to boost individual skills. ${ }^{1-3}$

There is a recognized need for sport-specific targeted training regimens and guidelines for coaches that help in developing desired characteristics in players. ${ }^{4}$ Plyometrics is one such popular training regimen that improves human performance. Though initially designed for athletes participating in the Olympics, the ability of plyometrics to augment muscle contractions in a specific pattern, subsequently generating powerful contractions, has gained popularity in sports such as field hockey. Plyometrics begins with an initial rapid muscle lengthening followed by a short rest phase and then a robust concentric muscle contraction. Enhancing speed-centred power is an additional benefit of plyometrics. ${ }^{5}$ Speed is one of the key ingredients during participation in any sport. Hockey players rely on quickness of movement which influences their performance during a game.

The unique physiological benefits of plyometric exercise has been explored by numerous research studies, most of which demonstrate the development of sport-specific skills such as agility, power, strength, and speed. $6-8$ Pre-stretch in the muscle tendon unit takes up the slack observed in the physiologic length-tension curve to enhance the force production of the muscles in the concentric phase of muscle contraction, thus augmenting the muscle power. ${ }^{9}$ The purpose of our study was to identify the effect of plyometrics on lower limb power, fatigue, and agility for male hockey players under age 16 . This study also aimed to determine the impact of detraining on these parameters. It was hypothesized that plyometric training would enhance the hockey player's lower limb power, fitness, and agility.

\section{METHODS}

This study was approved by the Institutional Review Board of a tertiary care hospital. Eight (8) schools of the Mumbai District were approached for this study. Fifty (50) elite male players (interschool and zonal champions) in the age group of 12 to 15 years voluntarily participated in this study. Written informed assent was obtained from each participant. Players who had not played field hockey for more than 3 months and players who had sustained trauma and major injuries were excluded from the study.

\section{Outcome Measures}

The outcome measures used were the vertical jump test, 40-metre sprint test, and shuttle cross pick up test.

\section{Vertical Jump Test}

The vertical jump test is designed to measure explosive leg power. ${ }^{10}$ To begin with the player stands sideways on to a wall and reaches up as high as he can with the hand touching the wall marking the standing reach height. He is then required jump as high as possible projecting the body upwards to achieve the jump height. Scoring: The distance between the standing reach height and the maximum jump height is measured. Best of the 3 attempts was considered (see Figure 1).
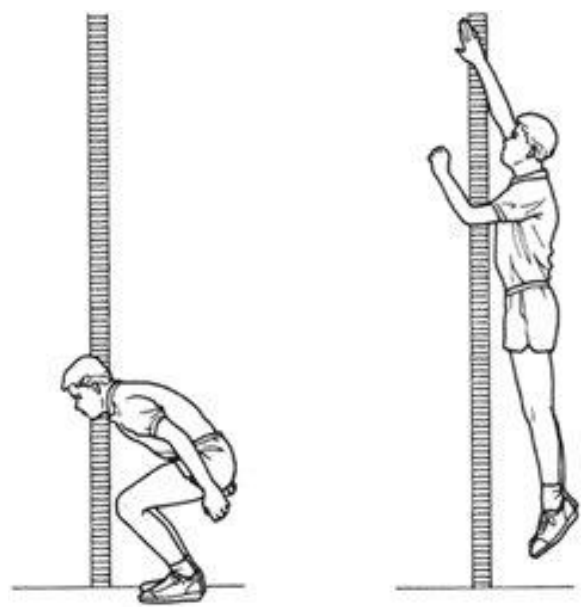

Figure 1: Vertical Jump Test (Courtesy: physioandrehab): Peak power is then calculated by Sayer's formula: PEAK POWER(WATTS)= 60.7*JUMP HEIGHT+ 45.3* BODY WEIGHT(KG) - 2055. 
40-Meter Sprint Test

The 40-metre sprint test was performed to determine the level of fitness. ${ }^{11}$ Fatigue is one of the factors that negatively affects the speed performance of an athlete. Repeated sprint tests, running-based anaerobic sprint tests, and repeated shuttle sprint ability tests have been used to judge the player performance and to estimate fatigue levels. Fatigue levels are calculated through various formulae indirectly either as percentage values or power output, but not many have used time differences. Unfortunately, some of these formulae used show low reliability. ${ }^{12-15}$ Fatigue is also influenced by many physical, physiological, emotional, and psychological factors, complicating the derivation of normal values. ${ }^{16}$

Field hockey is a dynamic sport involving many sprints, fast changes in direction with repeated sprints, and speed modification. Hence, fatigue valuation is used to assess player performance. Glaister et al stated that fatigue assessment was one of the main performance conclusions of the increasingly popular multiple sprint tests. ${ }^{12}$ In our study, we used the 40-meter sprint test to evaluate fatigue levels, with the measurement of time in seconds. The greater the time taken to complete the test, the greater is the fatigue levels of the player. After warmups for 10 minutes, the player sprints 40 metres as fast as possible repeatedly. A break of 30 seconds is given, and then the player completes the next sprint. Six such repeated sprints are performed after a pause of 30 seconds between each one. The time required for each sprint was documented separately using a stopwatch. Fatigue levels were calculated using the formula, fatigue level = total time for the six sprints minus the fastest time multiplied by $6 .{ }^{11}$

\section{Shuttle Cross Pickup Test}

The shuttle cross pick up test is a hockey specific hand-eye coordination and agility test. ${ }^{17}$ The player begins at the starting line $\mathrm{A}$. He then sprints to the centre cone $\mathrm{B}$ at 10 meters from cone $\mathrm{A}$. $\mathrm{A}$ ball is placed in the ring at position $\mathrm{C}$ and $\mathrm{E}$ which is 5 meters apart from centre cone $B$. From cone $B$, the player runs left around it to ring $C$ to pick up the ball. He then sprints back to the centre cone $B$ and then to the opposite ring $D$ to place the ball in the ring. He is then required to sprint back to the centre and then to ring $\mathrm{E}$, which is 5 meters ahead for the second ball pick-up, and finally sprints back through the start/finish line A with the second ball in their hand. The time taken to complete the test for two attempts is noted and the average of this is utilized for scoring (see Figure 2).

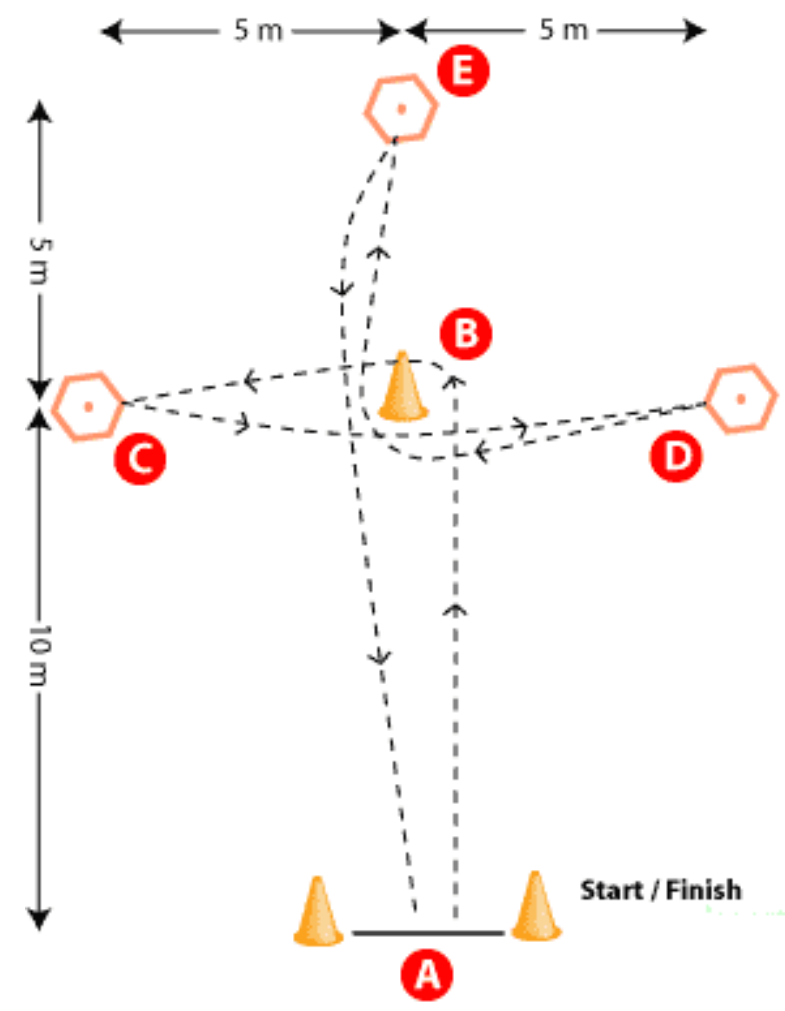

Figure 2: Shuttle Cross Pick up Test (Courtesy: Top end Sports)

After establishing a baseline, these parameters were reassessed at the end of the 3rd week, 6th week, and at the 8th week to determine the short term and post cessation effects of plyometric training. 


\section{The Plyometric Training Programme}

Plyometric training protocols in previous studies have used different durations of training. For example, Minj (5) used an 8week, $3 X$ per week protocol, while others like Singh et al used a protocol of 6-weeks, 2 X per week. ${ }^{5}$ Jastrzebski et al, Soumitra and Wondirad, and Campillo et al also used a 6-week follow up with two sessions per weeks. . $^{19-21}$ Looking at the effects of plyometric training from the reverse perspective, Meethal and Cheettiamkudiyil explored the effect of detraining after 2 weeks. ${ }^{22}$ Based on this literature, our study followed a protocol for 6-week of training at 3x per week followed by a 2-week detraining period. The training protocol included 5 specific plyometric exercises that were gradually increased in sets and repetitions over the 6-week period (see Table 1).

Table 1: Plyometric Protocol for the Players

\begin{tabular}{|c|c|c|}
\hline \hline EXERCISES & First 3 WEEKS & $4-6$ WEEKS \\
\hline Box jumps. & 3 sets 5 repetitions & 4 sets *5 repetitions \\
\hline Single leg box jumps. & 3 sets 5 repetitions & 4 sets *5 repetitions \\
\hline Depth jumps. & 2 sets *5 repetitions (each leg) & 3 sets*5 repetitions (each leg) \\
\hline Scissor jumps. & 3 sets ${ }^{*} 5$ repetitions & 4 sets *5 repetitions \\
\hline Side to side bounds. & 3 sets ${ }^{*}$ repetitions & 4 sets *5 repetitions \\
\hline
\end{tabular}

SPSS (Statistical Package for Social Sciences) version 24 was used for data analysis. The Shapiro-Wilk test was performed to verify the normality of the distribution. As the data was not normally distributed, statistical analysis was done using the nonparametric Friedman test. The Dunn's pairwise post hoc analysis was used to determine the statistical significance at $p$ value $<0.05$.

\section{RESULTS}

Table 2: Demographic Characteristics of the Athletes

\begin{tabular}{|c|c|}
\hline \hline Anthropometric Characteristics & Mean \pm Standard Deviation \\
\hline Age (years) & $12.78 \pm 0.88$ \\
\hline Weight (kg) & $44.4 \pm 11.46$ \\
\hline Height (metre) & $1.54 \pm 0.14$ \\
\hline BMl (kg/mt2) & $18.32 \pm 2.96$ \\
\hline
\end{tabular}

Table 3: Friedman Test Statistics of Lower Limb Power, Fatigue, and Agility Levels.

\begin{tabular}{|c|c|c|c|c|}
\hline Study Characteristics & & Mean Rank & $\begin{array}{l}\text { Chi- } \\
\text { square }\end{array}$ & $P$ value \\
\hline \multirow{4}{*}{ Lower limb power } & Baseline & 1.10 & \multirow{4}{*}{124.30} & \multirow{4}{*}{$<0.05$} \\
\hline & After 3 weeks & 2.89 & & \\
\hline & After 6 weeks & 3.87 & & \\
\hline & After 8 weeks & 2.14 & & \\
\hline \multirow{4}{*}{ Fatigue level } & Baseline & 2.85 & \multirow{4}{*}{118.56} & \multirow{4}{*}{$<0.05$} \\
\hline & After 3 weeks & 2.15 & & \\
\hline & After 6 weeks & 1.14 & & \\
\hline & After 8 weeks & 3.86 & & \\
\hline \multirow{4}{*}{ Agility level } & Baseline & 2.86 & \multirow{4}{*}{113.49} & \multirow{4}{*}{$<0.05$} \\
\hline & After 3 weeks & 2.16 & & \\
\hline & After 6 weeks & 1.16 & & \\
\hline & After 8 weeks & 3.82 & & \\
\hline
\end{tabular}

There was a statistically significant difference perceived in lower limb power, fatigue levels, and agility levels, suggesting that plyometric exercises caused a positive response in all the parameters tested. Dunn's pairwise post hoc analysis was done for 
lower limb power, fatigue levels and agility levels which revealed statistical significance throughout the 8-week plyometric protocol, suggesting that the increment in parameters begins as early as 3 weeks and continues till 8 weeks.

\section{DISCUSSION:}

Plyometric exercises were designed to counterbalance the gravitational forces on the musculoskeletal system. To produce greater force, it is essential to overcome the gravity and inertia generated by one's own body by use of strategies such as gathering for a throw, a jump, or a fast run.23-25 Plyometric training increases the proportion of force production (power) and conscription of motor units which augments the synchronisation of the firing frequency, as a result of the increase in excitability, sensitivity, and reactivity of the neuromuscular system. This enhances the motor learning and neuromuscular efficiency.

\section{Neurophysiology}

The basis for plyometric exercises is eccentric loading followed immediately by a concentric contraction. Neuromuscular adaptations to the stretch reflex, Golgi tendon organs, and changes in the muscle-tendon elasticity, pre-activation and potentiation are triggered by these exercises through the stretch shortening cycle (SSC).

The SSC encompasses three phases: the eccentric phase, the amortization phase, and the concentric phase. ${ }^{23-26}$ The eccentric phase is the first phase. Prior to muscle activation, this phase intensifies the muscle spindle activity by pre-stretching the muscle. The elastic properties of the muscle store the potential energy generated during this loading phase. Next comes the amortization phase, which is the electromechanical delay between the eccentric and concentric contraction. This phase involves dynamic stabilization and is the time between the end of the eccentric contraction and the initiation of the concentric contraction. Powerful response is generated on a prompt switch from an eccentric contraction to a concentric contraction. Last is the concentric (unloading) phase, which involves a concentric contraction, enhancing muscular performance following the eccentric phase of muscle contraction.

Our study demonstrates a significant response for the sport-specific parameters of plyometric training for six weeks, and also shows the attenuation of effects after two-weeks post cessation of training. Table 3 illustrates the statistics of the sport-specific parameters measured in this study at baseline, 3 weeks, 6 weeks, and 8 weeks. These values are statistically significant $(p<0.05)$.

\section{Effects of Plyometrics on Lower Limb Power}

Some of the mechanisms that contribute to improving the lower limb power include changes in the contractile apparatus of the muscle fibres, better neural adaptations such as muscle activation approaches, inter-muscular coordination, stretch reflex excitability, and changes in the structural design of the muscle (i.e. a decrease in fascicle angle and an increase in fascicle length of knee extensors), and in the elastic components of the muscle-tendon complex. ${ }^{27}$ These mechanisms are the results of the combined effects of enhanced neuromuscular control, a more reflexic central nervous system, and increased muscle spindle activity. Also, an eccentric contraction immediately following a concentric contraction increases the force generation because of the storage of potential elastic energy. ${ }^{28}$

The speed of muscular exertion is limited by neuromuscular coordination. The speed at which the muscular forces are generated for any activity determine the optimum reactive performance. Plyometric training improves the neuro muscular efficiency by enhancing the neuromuscular control of the contracting agonists and synergists, enabling the central nervous system to become more reflexic which then leads to an improvement in the reaction time. In plyometrics, maximum force production is generated by utilizing the elastic and proprioceptive properties of the muscles. This occurs as a result of stimulation of the mechanoreceptors leading to an increase in muscle recruitment in a minimal amount of time; thus, an increase in the muscle spindle activity occurs. Desensitization of the Golgi tendon organs increases the stimulation threshold for muscular inhibition, which increases force production. Similar improvement in the leg power, jump, and sprint performance was observed by Newton et al, Chelly et al, and Akdeniz and Sentürk, supporting the findings in our study. 29-31 Two weeks after training stopped, the power gained was still observed to be statistically significant; however, the values recorded were decreasing, suggesting that the increase in power achieved through six weeks was reversible. It should be noted, however, that this decrease in power was not to the former baseline values after only 2 weeks between the end of training and the measurements taken.

\section{Effect of Plyometrics on Fitness Level}

The speed and fatigue component are a measurement of the fitness level. 32,33 The time taken for sprinting was considerably reduced, suggestive of an increase in speed and decrease in fatigue level. This increase in the speed can be attributed to a more reflexic central nervous system -- an improved speed of muscle contraction and shorter amortization phase leading to enhanced performance of the hockey players. This, therefore, facilitates the neuromuscular system into making a more rapid transition from eccentric to concentric contraction. Probable mechanisms responsible for improving the sprint performance are preferential recruitment of fastest motor units, changes in temporal sequencing of muscle activation for more efficient 
movement or increased velocity of nerve conduction, or increased ability of the muscle to maintain recruitment and rapid firing throughout the sprint as hypothesised by Slimani et al. ${ }^{34}$ It is noted that the fatigue levels increased post cessation of training, but the fitness achieved is still statistically significant in this study.

\section{Effect of Plyometrics on Agility}

Sozbir speculated that improvements in agility were a result of enhanced motor unit recruitment patters or neural adaptations..$^{35}$ Neural adaptations include synchronous firing of motor neurons, or better neural impulses from the spinal cord, or increased neuromuscular coordination as a result of improved coordination between the CNS signal and proprioceptive feedback. Agility was seen to improve as a result of a decrease in the ground reaction time with an increase in muscle power and efficient movement ${ }^{36}$ Similar results were found in a study done by Singh et al which showed a significant improvement in agility, vertical jump height, and peak torque ratio as a result of plyometric training. ${ }^{37}$ As with power and fatigue levels, the post cessation agility levels were also observed to decrease though not completely reversed.

Based on changes to these parameters, we can theorize that two weeks' termination of plyometric training is probably not enough to reverse all the positive effects gained. Field hockey is a lively sport with constantly changing player dynamics from defense to offense. This 6-weeks plyometric training proved to be short and effective showing significant improvement in power, agility, and overall fitness of the athletes. The effects of plyometric training demonstrated a decreasing trend post 2week training cessation but still effective and not to baseline values. Thus, plyometrics should be considered while planning and designing sport-specific targeted training.

\section{CONCLUSION}

The six-week plyometric training regimen resulted in significant enhancement of all the test parameters, including power, fitness, and agility, with a visible effect from the third week onward. Because these are the basic parameters that are determinants of not only the sports performance but also general well-being, health professionals and coaches should include plyometric training as a part of their regular drills for targeted advancement of the players. This study also testified that the training effects last beyond the two-week post cessation of the protocol.

\section{REFERENCES}

1. Sharma HB, Kailashiya J. Effects of six week sprint-strength and agility training on body composition, cardiovascular, and physiological parameters of male field hockey players. J Strength Cond Res. 2018;32(4):894-901.

2. Manna I, Khanna GK, Dhara PC. Effect of training on anthropometric, physiological and biochemical variables of elite field hockey players. International Journal of Sports Science and Engineering. 2010;4:229-238.

3. Hanson ED, Srivatsan SR, Agrawal S, Menon KS, Delmonico MJ, Wang MQ, and Hurley BF. Effects of strength training on physical function: influence of power, strength, and body composition. J Strength Cond Res. 2009;23:26272637.

4. Melton DI, Katula JA, and Mustian KM. The current state of personal training: a 12-industry perspective of personal trainers in a small Southeast community. J Strength Cond Res. 2008;22:883-889.

5. Minj A. Effect of 8 weeks plyometric training on speed of hockey players. International Journal of Behavioural Social and Movement Sciences. 2015;4(1):10-15.

6. Miller MG, Herniman JJ, Ricard MD, Cheatham CC, Michael TJ. The effects of a 6-week plyometric training program on agility. J.Sports Sci Med. 2006;5(3):459-465.

7. Vaczi M, Tollar J, Meszler B, Juhasz, I, Karsai, I. Short-term high intensity plyometric training program improves strength, power, and agility in male soccer players. J. Hum. Kinet. 2013;36,17-26.

8. Fernandez-Fernandez J, Saez de Villarreal E, Sanz-Rivas D, Moya M. The effects of 8-week plyometric training on physical performance in young tennis players. Pediatr Exerc Sci. 2016;28,77-86.

9. Davies G, Riemann BL, Manske, R. Current concepts of plyometric exercise. Int J Sports Phys Ther. 2015;10(6): 760786

10. Robert Wood, First Published: (2008), Page Title: Vertical Jump Test, Website Name: Top end Sports, Webpage URL: http://www.topendsports.com/testing/tests/vertjump.html.

11. Mackenzie, B. 40-Metre Multiple Sprint Test. 2006. Available from: https://www.brianmac.co.uk/40sprint.htm

12. Glaister M, Howatson G, Pattison JR, McInnes $G$. The reliability and validity of fatigue measures during multiple sprint work: an issue revisited. J Strength Cond Res. 2008;22(5):1597-1601.

13. Pavlovic R, Idrizovic K, Bosnjak G, Pupis M. Fatigue index - indicator of anaerobic abilities students. International Journal of Science Culture and Sport. 2016;4(3):315-325

14. Impellizzeri FM, Rampinini E, Castagna C, Bishop D, Ferrari Bravo D, Tibaudi A, Wisloff U. Validity of a repeated sprint test for football. Int J Sports Med. 2008;29:899-905.

15. Hazir T, Kin Isler A, Kadioglu M, Unver E. Reliability of performance outputs and formulas related with fatigue index in 10 X 20 m repeated sprint test. Turk J Sports Med. 2019;54(4):267-275. 
16. Schiphof-Godart L, Roelands B, Hettinga FJ. Drive in sports: How mental fatigue affects endurance performance (Perspective Article). Front.Psychol. 2018; 9:1383; 1-7.

17. Robert Wood, First Published: (2008), Page Title: Field Hockey Fitness Testing, Website Name: Top end Sports, Webpage: urlhttp://www.topendsports.com/sport/hockey/testing.

18. Singh J, Appleby BB, Lavender AP. Effect of plyometric training on speed and change of direction ability in elite field hockey players. Sports. 2018;6(144): 1-14.

19. Jastrzebski Z, Wnorowski K, Mikolajewski R, Jaskulska E, Radziminski L. (2014) The effect of a 6-week plyometric training on explosive power in volleyball players. BJHPA. 2014;6(2): 79-89.

20. Mondal S, Wondirad S. (2014) Effect of 6-week plyometric training on vertical jump performance of Maychew Athletes. IJPEHSS. 2014, 3(1): 1-6.

21. Ramirez-Campillo $R$, Andrade $D$ and Izquierdo $M$. Effects of plyometric training volume and training surface on explosive strength. J Strength Cond Res. 2012;27(10):2714-2722.

22. Meethal $A$, Chettiamkudiyil $H B$. Effect of plyometric training and $S A Q$ training followed by detraining on agility of intercollegiate level footballers. International Journal of Physical Education, Fitness, and Sports. 2015;4(2): 22-31

23. Hansen D, Kennelley S (2017). Plyometric Anatomy-Human Kinetics. Chapter 1, 2 and 3. 2017. ISBN 978-4925-3349-8

24. Verma C, Subramanium L, Krishnan V. Effect of plyometric training on vertical jump height in high school basketball players: A randomised control trial. Int J Med Res Health Sci. 2015;4(1):7-12

25. Clark M, Lucett S, Kirkendall DT. NASM's Essentials of Sports Performance Training. 2010. Philadelphia: Wolters Kluwer/Lippincott Williams \& Wilkins, Chapter 8, 207-226.

26. Fukutani A, Kurihara T, Isaka T. Factors of force potentiation induced by stretch shortening cycle in plantarflexors. PLoS One. 2015;10(6): e0120579.

27. Moran JJ, Sandercock GR, Ramírez-Campillo R, Meylan CM, Collison JA, Parry DA. Age-related variation in male youth athletes' countermovement jump following plyometric training: a meta-analysis of controlled trials. J Strength Cond Res. 2017;31(2):552-565

28. Asadi A. Muscular performance adaptations to short-term plyometric training on sand: influence of interday rest. $J$. Hum. Sport Exerc. 2015;10 (3):775-784

29. Newton RU, Kraemer WJ, Häkkinen K. Effects of ballistic training on preseason preparation of elite volleyball players. J. Med Sci Sports Exerc. 1999;31:323-30.

30. Chelly M, Ghenem M, Khalil A, Hermassi S, Tabka Z, Shephard R. Effects of in-season short-term plyometric training program on leg power, jump- and sprint performance of soccer players. J Strength Cond Res. 2010 Oct; 24(10): 26702676

31. Akdeniz A, Sentürk A. Reviewing the effect of plyometric training performed by icemen play in super league on quick power and maximal power. European Journal of Physical Education and Sport Science. 2018;4(2): $37-51$

32. Elferink-Gemser MT, Visscher C, Van Duijn MA, and Lemmink KA. Development of the interval endurance capacity in elite and sub-elite youth field hockey players. Br J Sports Med. 2006;40(4): 340-345.

33. Kenney WL, Wilmore JH, and Costill DL . Adaptations to Aerobic and Anaerobic Training., in: Physiology of Sports and Exercise. Champaign (IL): Human Kinetics, @ 2012;pp 247-278.

34. Slimani M, Chamari K, Miarka B. Effects of plyometric training on physical fitness in team sport athletes: a systematic review. J Hum Kinet. 2016;53: 231-247

35. Sozbir K. Effects of 6-week plyometric training on vertical jump performance and muscle activation of lower extremity muscles. The Sport Journal, 2016;21;ISSN:1543-9518.

36. Potteiger A., Lockwood H., Haub D, Dolezal, Brett A., Almuzaini, Khalid S., Schroeder, Jan m., et al. Muscle power and fibre characteristic following 8 weeks of plyometric training. Journal of Strength and Conditioning Research. 1999;13: 275- 279

37. Singh A, Boyat AK, Sandhu JS. (2015). Effect of a 6 week plyometric training program on agility, vertical jump height and peak torque ratio of Indian taekwondo players, Sport Exerc Med Open J. 2015;1(2): 42-46. 